\title{
Hodgkin's lymphoma as a rare variant of Richter's transformation in chronic lymphocytic leukemia: A case report and review of the literature
}

\author{
SNJEZANA JANJETOVIC ${ }^{1}$, HEINZ-WOLFRAM BERND ${ }^{2}$, CARSTEN BOKEMEYER $^{1}$ and WALTER FIEDLER ${ }^{1}$ \\ ${ }^{1}$ Department of Oncology and Hematology, BMT with Section of Pneumology, Hubertus Wald Tumorzentrum, \\ University Cancer Center Hamburg, University Medical Center Hamburg-Eppendorf, 20246 Hamburg; \\ ${ }^{2}$ Department of Pathology, University Hospital of Schleswig-Holstein, 23562 Campus Lübeck, Germany
}

Received May 22, 2015; Accepted October 29, 2015

DOI: $10.3892 / \operatorname{mco} .2016 .727$

\begin{abstract}
Richter's transformation induces an aggressive clinical course in chronic lymphocytic leukemia (CLL). In the majority of cases, Richter's transformation manifests itself as a high-grade B-cell non-Hodgkin's lymphoma (B-NHL). However, other histological types, such as classical Hodgkin lymphoma (cHL), lymphoblastic lymphoma, hairy cell leukemia and high-grade T-cell NHL have been described previously. The present study reports a rare case of CLL with transformation into classical Hodgkin's lymphoma (cHL). The common clonal origin of CLL and cHL was documented by immunoglobulin gene rearrangement analysis performed using multiplex polymerase chain reaction. Following a review of the literature, treatment of secondary Hodgkin's lymphoma is discussed, and prognosis is often poor.
\end{abstract}

\section{Introduction}

Richter's transformation represents an aggressive evolution of chronic lymphocytic leukemia (CLL) (1). High-grade B-cell non-Hodgkin lymphoma (B-NHL) is the most common histological type of Richter's transformation (2). However, other histological types, such as classical Hodgkin lymphoma (cHL) (2-4), lymphoblastic lymphoma (5), hairy cell leukemia (6) and high-grade T-cell NHL $(7,8)$ have been reported previously. B-CLL and cHL can be clonally related or independent lymphomas $(9,10)$.

Different therapy modalities are reviewed in the present study regarding the optimal treatment strategy for Hodgkin

Correspondence to: Mrs. Snjezana Janjetovic, Department of Oncology and Hematology, BMT with Section of Pneumology, Hubertus Wald Tumorzentrum, University Cancer Center Hamburg, University Medical Center Hamburg-Eppendorf, Martinistrasse 52, 20246 Hamburg, Germany

E-mail: s.janjetovic@uke.de

Key words: Richter transformation, chronic lymphocytic leukemia transformation. However, despite the different chemotherapeutic regiments, the prognosis remains poor compared to de novo Hodgkin's lymphoma (4) and new therapeutic approaches shoud be considered in the future.

\section{Case report}

A 70-year-old male presented with right thoracic pain. A computer tomography (CT) scan showed thoracic and retroperitoneal lymphadenopathy with a maximal diameter of $21 \mathrm{~mm}$, and a pleural effusion at the right side. Routine blood investigations showed normal erythrocyte, platelet and leukocyte counts.

The cytology of the pleural effusion revealed an atypical B-cell population consistent with B-CLL (Fig. 1). Bone marrow aspiration confirmed the diagnosis of CLL, stage Binet B. Fluorescence in situ hybridisation (FISH) analyses of bone marrow excluded trisomy 12, and deletions of 11q22.3, 13q14 and $17 \mathrm{p} 13$. FISH experiments were performed using commercially available probes from Abbott (Abbott Park, IL, USA), according to the manufacturer's protocol. A watch-and-wait approach was applied. During the following months the patient suffered from repeated infections due to hypogammaglobulinemia with immunoglobulin $\mathrm{G}(\mathrm{IgG})<3 \mathrm{~g} / \mathrm{l}$, and therefore, immunoglobulin substitution and antibiotic therapy was repeatedly necessary.

Two months later the patient complained of fever, cough, right thoracic pain and fatigue. A repeated CT scan of the thorax showed a pleural effusion again. In order to exclude pleural empyema, video-assisted thoracoscopic surgery with pleurectomy was performed. Histological analysis revealed a marked pleural fibrosis with scattered cellular lymphoid nodules. The latter contained a variable mixture of small Band T-cell lymphocytes, histiocytes, fibroblasts, eosinophils and scattered atypical blasts corresponding to Hodgkin cells and Reed-Sternberg (HRS) cells. The B-cell lymphocytes were cluster of differentiation (CD) 20-positive, but did not co-express CD5 or CD23, as assessed using monoclonal antibodies recognizing these antigens in paraffin-embedded tissue. Therefore, the small aggregates of the B-cells did not fulfil the diagnostic criteria for a B-CLL infiltration. HRS-blasts proved 
Table I. Most common described treatment options of Hodgkin's transformation of chronic lymphocytic leukemia $(4,11)$.

Treatment Specific drugs and therapy

ABVD
CVPP \pm involved field radiation
MOPP
CHOP \pm R
FCR
Other

ABVD

CVPP \pm involved field radiation

MOPP

FCR

Doxorubicin, bleomycin, vinblastine and dacarbazine
Cyclophosphamide, vinblastine, procarbazine and prednisone \pm involved field radiation
Mecholrethamine, oncovin, procarbazine and prednisone
Cyclophosphamide, doxorubicin, vincristine and prednisone \pm rituximab
Fludarabine, cyclophosphamide and rituximab
Mitoxantrone, vincristine, vinblastine and prednisone; rituximab; or cidofovir

Doxorubicin, bleomycin, vinblastine and dacarbazine

Cyclophosphamide, vinblastine, procarbazine and prednisone \pm involved field radiation Mecholrethamine, oncovin, procarbazine and prednisone

Cyclophosphamide, doxorubicin, vincristine and prednisone \pm rituximab

Fludarabine, cyclophosphamide and rituximab

to be CD20-negative, but showed expression of CD30, CD15 and PAX5. Additionally, in situ hybridization for Ebstein-Barr virus (EBV) was positive. Accordingly, diagnosis of nodular sclerosis-type classical Hodgkin's lymphoma was rendered. The disease was staged as IV due to the pleural involvement.

Comparative molecular analyses of the $\mathrm{IgH}$ of selected tissue compartments of the pleural biopsy and of peripheral blood B-lymphocytes were performed using multiplex polymerase chain reaction (PCR) with BIOMED-2 primer sets, as previously described (11). The resulting PCR amplicons showed identical sizes indicating the same $\operatorname{IgH}$ heavy chain rearrangement in both lymphoma manifestations, and thus a common clonal origin of CLL and cHL.

Chemotherapy with $25 \mathrm{mg} / \mathrm{m}^{2}$ doxorubicin, $10 \mathrm{mg} / \mathrm{m}^{2}$ bleomycine, $6 \mathrm{mg} / \mathrm{m}^{2}$ vinblastine and $375 \mathrm{mg} / \mathrm{m}^{2}$ dacarbazine on days 1 and 15 was introduced, for a total of 8 cycles. A complete remission was achieved.

\section{Discussion}

Approximately $2-8 \%$ of all patients diagnosed with CLL transform into more aggressive lymphoma known as Richter's syndrome (1). The most common is the transformation of CLL into a high-grade B-NHL (2). Transformation to HL is thought to occur in $\sim 0.4 \%$ of all CLL patients $(3,4)$. Other histological types of Richter's transformation have also been described, including lymphoblastic lymphoma (5), hairy cell leukemia (6) and high-grade T-cell NHL $(7,8)$.

A published small series suggested that tumor cells in B-NHL and cHL can be clonally related to B-CLL clone or arise as an independent, secondary lymphoma $(9,10)$. Additionally, it has been postulated that immunosuppressive therapy in CLL, particularly fludarabin, may increase the risk of Richter's transformation to cHL $(12,13)$.

EBV infection is only infrequently detected in CLL by conventional diagnostic approaches. However, it has been shown that EBV persistence in the lymphocytes of patients with CLL may lead to the more aggressive disease and Richter's transformation into cHL $(14,15)$. Several studies showed that EBV small non-coding RNA (EBERs) expressed in latently infected cells have a critical role in B-cell transformation and induction of resistance to apoptosis, which may lead to CLL progression (16).

According to the largest series of published Hodgkin transformation in CLL, in 3 out of 4 analysed patients $(75 \%)$, EBV was detected (4). In the patient reported in the present study, Hodgkin cells were also EBV-positive, as documented

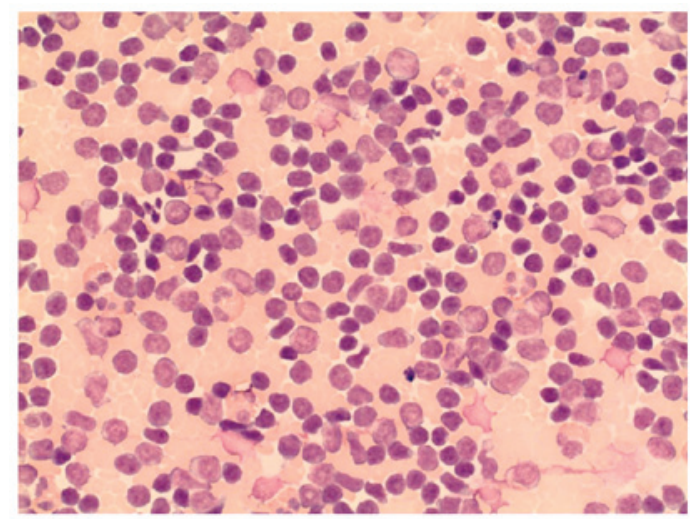

Figure 1. Cytology of the pleural effusion revealing an atypical B-cell population, consistent with B-chronic lymphocytic leukemia. therapy in the treatment of Hodgkin transformation in CLL remains to be elucidated.

Bone marrow cytogenetics revealed a normal karyotype in $42 \%$ of the cases in one study (4), wheres abnormalities, including -Y, 11q-, del (13), t(9;15), trisomy 12, -11 and -17, were identified in $<40 \%$ of the cases (4). FISH analyses of the bone marrow in the present patient were negative for trisomy 12 , deletion 11q22.3, deletion $13 q 14$ or deletion $17 \mathrm{p} 13$.

cHL transformation in CLL has a poor outcome compared to the de novo $\mathrm{Chl}$ (4). The administration of different therapy modalities to the patients with HL as Richter's transformation has been described previously (Table I). However, the median overall survival, according to the largest series of published Hodgkin transformation in CLL, was only 8 months (4).

More effective treatment is required for patients who develop Hodgkin transformation of CLL. The role of high-dose chemotherapy with autologous stem cell transplantation in these patients is not well defined. However, this more aggressive approach may not be feasible in elderly groups of patients.

\section{References}

1. Rossi D, Cerri M, Capello D, Deambrogi C, Rossi FM, Zucchetto A, De Paoli L, Cresta S, Rasi S, Spina V, et al: Biological and clinical risk factors of chronic lymphocytic leukaemia transformation to Richter syndrome. Br J Haematol 142: 202-215, 2008.

2. Tsimberidou AM and Keating MJ: Richter syndrome: Biology, incidence and therapeutic strategies. Cancer 103: 216-228, 2005. by EBER in situ hybridisation. However, the role of antiviral 
3. Nemets A, Ben Dor D, Barry T, Ducach A, Blumental R, Ben Alon D and Lugassy G: Variant Richter's syndrome: A rare case of classical Hodgkin's lymphoma developing in a patient with chronic lymphocytic leukemia treated with fludarabine. Leuk Lymphoma 44: 2151-2154, 2003.

4. Tsimberidou AM, O'Brien S, Kantarjian HM, Koller C, Hagemeister FB, Fayad L, Lerner S, Bueso-Ramos CE and Keating MJ: Hodgkin transformation of chronic lymphocytic leukemia: The M. D. Anderson cancer center experience. Cancer 107: 1294-1302, 2006.

5. Pistoia V, Roncella S, Di Celle PF, Sessarego M, Cutrona G, Cerruti G, Boccaccio GP, Grossi CE, Foà R and Ferrarini M: Emergence of a B-cell lymphoblastic lymphoma in a patient with B-cell chronic lymphocytic leukemia: Evidence for the single-cell origin of the two tumors. Blood 78: 797-804, 1991.

6. Duchayne E, Delsol G, Kuhlein E, Klein B, Zhang XG, Attal M Cassard G and Laurent G: Hairy cell transformation of a B-cell chronic lymphocytic leukemia: A morphological, cytochemical, phenotypic and molecular study. Leukemia 5: 150-155, 1991.

7. Strickler JG, Amsden TW and Kurtin PJ: Small B-cell lymphoid neoplasms with coexisting T-cell lymphomas. Am J Clin Pathol 98: 424-429, 1992.

8. Lee A, Skelly ME, Kingma DW and Medeiros LJ: B-cell chronic lymphocytic leukemia followed by high grade T-cell lymphoma. An unusual variant of Richter's syndrome. Am J Clin Pathol 103: 348-352, 1995

9. Mao Z, Quintanilla-Martinez L, Raffeld M, Richter M, Krugmann J, Burek C, Hartmann E, Rudiger T, Jaffe ES, Müller-Hermelink HK, et al: IgVH mutational status and clonality analysis of Richter's transformation: Diffuse large B-cell lymphoma and Hodgkin lymphoma in association with B-cell chronic lymphocytic leukemia (B-CLL) represent 2 different pathways of disease evolution. Am J Surg Pathol 31: 1605-1614, 2007.
10. van den Berg A, Maggio E, Rust R, Kooistra K, Diepstra A and Poppema S: Clonal relation in a case of CLL, ALCL and Hodgkin composite lymphoma. Blood 100: 1425-1429, 2002.

11. van Dongen JJ, Langerak AW, Brüggemann M, Evans PA, Hummel M, Lavender FL, Delabesse E, Davi F, Schuuring E, et al: Design and standardization of PCR primers and protocols for detection of clonal immunoglobulin and T-cell receptor gene recombinations in suspect lymphoproliferations: report of the BIOMED-2 Concerted Action BMH4-CT98-3936. Leukemia 17: 2257-2317, 2003

12. Bockorny B, Codreanu I and Dasanu CA: Hodgkin lymphoma as Richter transformation in chronic lymphocytic leukaemia: A retrospective analysis of world literature. Br J Haematol 156: 50-66, 2012.

13. Dasanu CA: Intrinsic and treatment-related immune alterations in chronic lymphocytic leukaemia and their impact for clinical practice. Expert Opin Pharmacother 9: 1481-1494, 2008.

14. Dolcetti R and Carbone A: Epstein-Barr virus infection and chronic lymphocytic leukemia: A possible progression factor? Infect Agent Cancer 5: 22, 2010.

15. Rubin D, Hudnall SD, Aisenberg A, Jacobson JO and Harris NL Richter's transformation of chronic lymphocytic leukemia with Hodgkin's-like cells is associated with Epstein-Barr virus infection. Mod Pathol 7: 91-98, 1994.

16. Iwakiri D and Takada K: Role of EBERs in the pathogenesis of EBV infection. Adv Cancer Res 107: 119-136, 2010 